\title{
MISINTERPRETATION OF THE 'SAFE SEX/NO SEX' PREVENTION STRATEGY
}

To the Editor: We refer to the letter to the editor by MacPhail et al. ${ }^{1}$ discussing the specifics of Whiteside and Parkhurst's article in the April 2010 issue of the Journal. ${ }^{2}$ MacPhail et al. reported that they theoretically agreed with Whiteside and Parkhurst that refraining from sex during the acute HIV infection period might reduce the rate of HIV transmission when implemented on a wide scale. ${ }^{1}$ They summarised the scientific logic of the 'safe sex/no sex' prevention strategy, and explained what the acute HIV infection period is and how critical it is in the transmission of HIV.

MacPhail and her colleagues showed interest in the 'safe sex/no sex' behavioural intervention and its potential significant contribution to global prevention efforts. However, they misrepresent the core arguments Whiteside and Parkhurst propose. In their letter they present the 'safe sex/no sex' strategy incompletely. For example, they report that 'The authors suggest that a limited period of population-wide sexual abstinence might be an effective and low-cost method of interrupting the transmission of HIV' and that 'a limited period of abstinence might be theoretically infective in limiting HIV transmission', suggesting that the strategy focuses solely on abstinence. While an important aspect of the strategy, abstinence is not the entire approach, and indeed the benefits of a month-long commitment to 'safe sex' behaviour should not be disregarded owing to the perceived infeasibility of a month-long commitment to 'no sex'.

Along with considering a limited period of abstinence, Whiteside and Parkhurst promote 'safe sex' or sexual activity engaged in by people who have taken precautions to protect themselves against HIV infection, for instance by adhering to correct and consistent condom use, reducing concurrency, and promoting circumcision and microbicide gel use and other HIV prevention measures. The key arguments for the 'safe sex/no sex' prevention strategy are therefore not completely expressed, being reduced to just abstinence. Whiteside and Parkhurst's article clearly elucidated that the potential intervention would be an aggressive national campaign to ensure that everyone who is sexually active in a population, whether HIV positive or negative, either commit to $100 \%$ condom use or refrain from sexual intercourse over a period of a month or longer. ${ }^{2}$

MacPhail et al. reported on research with 37 individuals in Lilongwe, Malawi, and Johannesburg, South Africa, to test this theory. Their research tested the 'no sex' and 'safe sex' aspects of the proposed prevention strategy as two distinct and potential interventions to interrupt HIV transmission during the acute infection period. As a result, their findings that there was limited support for the strategy in a population of individuals with known HIV infection, and that there is likely to be even less support from individuals who do not know their status or do not perceive themselves to be at risk of HIV infection, do not adequately indicate the potential challenges the 'safe sex/no sex' prevention strategy is likely to encounter, as the study investigated 'safe sex' and 'no sex' as different interventions, not one as proposed in the 'safe sex/no sex' prevention strategy. This does not mean that MacPhail et al.'s research is not important - it will help to articulate the difficulties with a straight 'no sex' approach to the intervention, as well as pointing towards other potential barriers. It does not invalidate the intervention strategy, and perhaps even suggests the need to test out a strategy that is focused on both abstinence and safe sex.

We have reason to believe that, while difficult, an intervention that focuses on promoting both 'safe sex' and 'no sex' has the potential to be successful. In a recent qualitative study of the 'conceptual impact' of this strategy, we found that most of the participants (members of non-governmental organisations (NGOs), academia, the Department of Health, the media and HIVIAIDS researchers) were in favour of the 'safe sex/ no sex' prevention strategy (unpublished data). The great majority of the positive respondents reported that it should be implemented because it focuses on both infected and uninfected individuals without necessarily requiring people to know their HIV status. The concern of many participants was the personal or individual willingness and commitment of both infected and uninfected individuals to abstain or engage in safe sex, and not the support they would get in the population to abstain or engage in safe sex, as reported by MacPhail et al. ${ }^{1}$ In our study, a handful of participants, 2 out of 4 , were not in favour of the idea that reported that the 'safe sex/no sex' prevention strategy would not work due to lack of interpersonal support in the population. ${ }^{3}$

In our study, participants in favour of the 'safe sex/ no sex' prevention strategy believed that it would uphold and promote rights of privacy of individuals and therefore cause less stigma and discrimination based on HIV status. Participants explained that this would make it easy to mobilise individuals and communities to abstain from sex or engage in safe sex, as it can be done without distinction of whether one is HIV-positive or negative. However, organisers of the prevention strategy 
would be aware of the HIV status of the populations as this would help them to monitor the average HIV viral load in the population before, during and after the period of abstinence and safe sex to see how much it impacted on infectiousness, and to get better estimates of effectiveness in practices. The argument by MacPhail et al. that the 'safe sex/no sex' prevention strategy may have less support from individuals because they did not know their status or perceived that they were not at risk of HIV infection ${ }^{1}$ was not reported as a barrier (in our study) to the feasibility and acceptability of the 'safe sex/no sex' prevention strategy. ${ }^{3}$ This is attributed to the fact that the study investigated the feasibility and acceptability of both 'safe sex' and 'no sex' as one strategy, implied by the 'safe sex/no sex' prevention strategy championed by Whiteside and Parkhurst.

As we have found that in theory there is wide support for this prevention strategy (including support by MacPhail et al.), it would be of benefit to the entire HIVIAIDS research community for it to be properly articulated and debated. To reduce the strategy to a period of abstinence, as MacPhail et al.'s letter to the editor did, obscures the proposed strategy and may prevent us from properly engaging with a very promising prevention effort.

\section{G Mutinta}

\section{A Whiteside}

Health Economics and HIV/AIDS Research Division, University of KwaZulu-Natal, Durban

\section{REFERENCES}

1. MacPhail C, Pettifor A, Corneli A. Feasibility and acceptability of sexual abstinence for interruption of HIV transmission among individuals with acute infection - formative data from CHAVI 011. Southern African Journal of HIV Medicine 2011;12(2):46.

2. Whiteside A, Parkhurst JO. Innovative Responses for preventing HIV transmission: The protective value of population-wide interruptions of risk activity. Southern African Journal of HIV Infection 2010;11(1):19-21.

3. Mutinta G, McAlister H, Ga'al K. An explorative study on the 'conceptual impact' of the 'safe sex/no sex' HIV prevention strategy. African Journal for HIV Research (in press). 


\section{2 peas in one pod... Introducing}

\section{Aspen Tenofovir \& Emtricitabine}

A fixed dose combination of $300 \mathrm{mg}$ tenofovir disoproxil fumarate \& $200 \mathrm{mg}$ emtricitabine
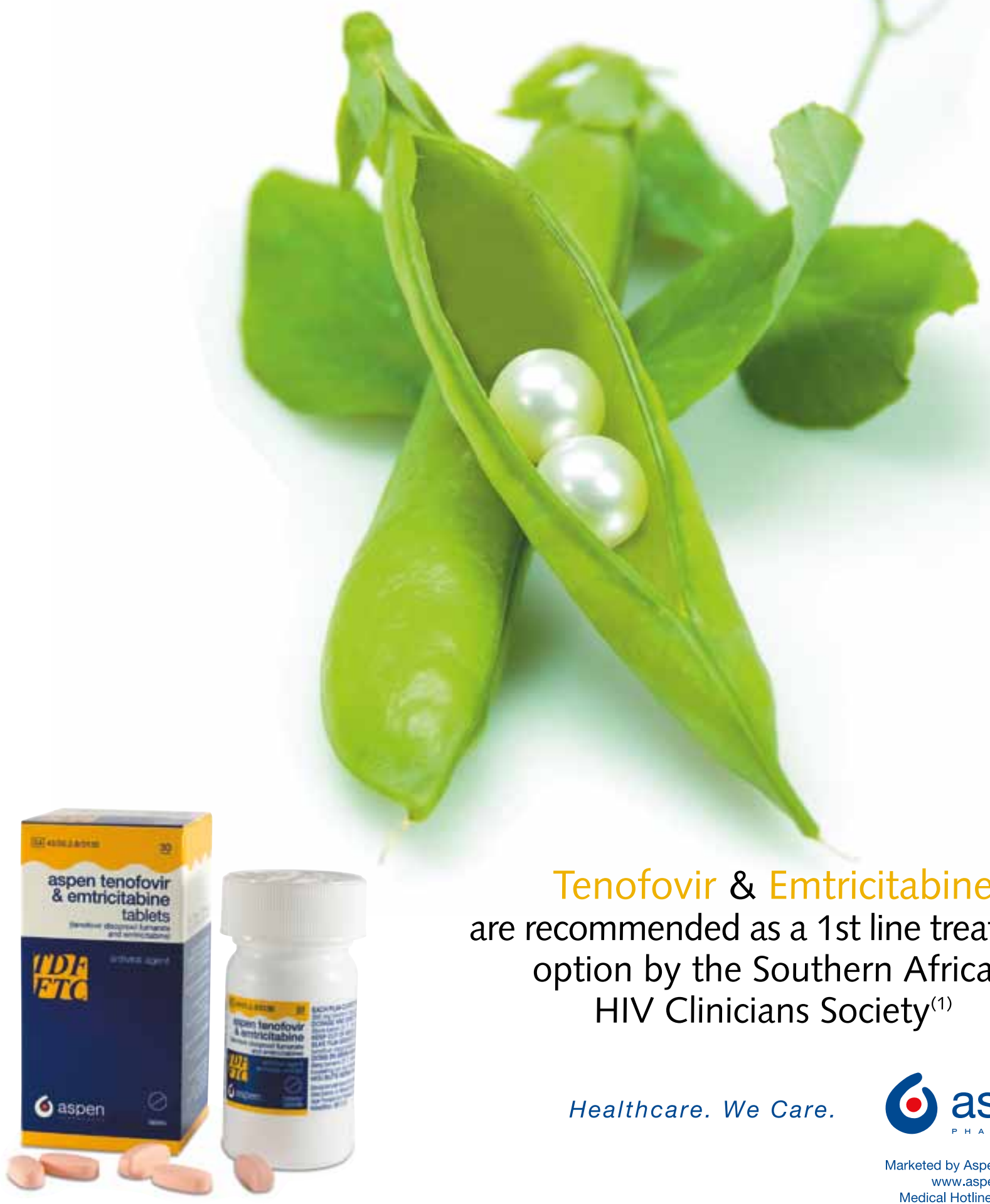

\section{Tenofovir \& Emtricitabine are recommended as a 1 st line treatment option by the Southern African HIV Clinicians Society ${ }^{(1)}$}

Healthcare. We Care.

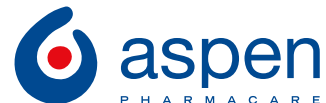

Marketed by Aspen Pharmacare www.aspenpharma.com Medical Hotline 0800118088

WARNING: LACTIC ACIDOSIS AND SEVERE HEPATOMEGALY WITH STEATOSIS, INCLUDING FATAL CASES, HAVE BEEN REPORTED WITH THE USE OF NUCLEOSIDE ANALOGUES ALONE OR IN SAFETY AND EFFICACY OF ASPEN TENOFOVIR \& EMTRICITABINE HAS NOT BEEN ESTABLISHED IN PATIENTS CO-INFECTED WITH HBV AND HIV. SEVERE ACUTE EXACERBATIONS OF HEPATITIS B HAVE BEEN REPORT LABORATORY FOLLOW-UP FOR AT LEAST SEVERAL MONTHS IN PATIENTS WHO DISCONTINUE ASPEN TENOFOVIR \& EMTRICITABINE AND ARE CO-INFECTED WITH HIV AND HBV. IF APPROPRIATE, INITIATION OF ANTI-HEPATITIS B THERAPY MAY BE WARRANTED.

Reference: 1. Guidelines. Antiretroviral Therapy in Adults. The Southern African Journal of HIV Medicine 2008; 18-31.

S4 Aspen Tenofovir \& Emtricitabine. Reg. No: 43/20.2.8/0130. Each film-coated tablet contains $300 \mathrm{mg}$ tenofovir disoproxil fumarate which is equivalent to $245 \mathrm{mg}$ of tenofovir disoproxil and $200 \mathrm{mg}$ emtricitabine.

For full prescribing information refer to the package insert approved by the medicines regulatory authority.

Marketed by Aspen Pharmacare for Pharmacare Limited, Co. Reg. No.: 1898/000252/06. Building 12, Healthcare Park, Woodlands Drive, Woodmead 2191. Tel (011) 239 3400, Fax (011) 239 3438. www.aspenpharma.com Medical Information Hotline 0800118 088. A12533 12/10. 
A Breakth


*For full prescribing information, please refer to the package insert approved by the medicines regulatory authority.

S4 INTELENCETM Tablets. Reg. No. 43/20.2.8/0780. Each tablet contains $100 \mathrm{mg}$ of etravirine. Excipients include: lactose monohydrate, hypromellose, microcrystalline cellulose, colloidal anhydrous silica, croscarmellose sodium, magnesium stearate. Marketed under license from Tibotec Pharmaceutica
12, Healthcare Park, Woodlands Drive, Woodmead 2191. Tel (011) 239 3400, Fax (011) 239 3438. A13748 10/11.

\section{6 aspen Heattcare. We care.}

For the treatment of HIV-1 infection in ARV treatment-experienced adult patients with NNRTI resistance*

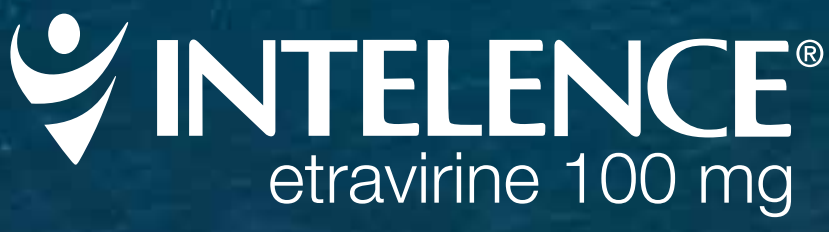

ANEXT GENERATION NAB 